\title{
Prenormative verification and validation of a protocol for measuring magnetite- maghemite ratios in magnetic nanoparticles
}

Bogart, Lara K.; Fock, Jeppe; da Costa, Geraldo M; Witte, Kerstin; Greneche, Jean-Marc; Zukrowski, Jan; Sikora, Marcin; Latta, Drew E.; Scherer, Michelle M.; Hansen, Mikkel Fougt

Total number of authors:

12

Published in:

Metrologia

Link to article, DOI:

$10.1088 / 1681-7575 / a c 36 b 6$

Publication date:

2022

Document Version

Publisher's PDF, also known as Version of record

Link back to DTU Orbit

Citation (APA):

Bogart, L. K., Fock, J., da Costa, G. M., Witte, K., Greneche, J-M., Zukrowski, J., Sikora, M., Latta, D. E., Scherer, M. M., Hansen, M. F., Frandsen, C., \& London, U. (2022). Prenormative verification and validation of a protocol for measuring magnetite-maghemite ratios in magnetic nanoparticles. Metrologia, 59(1), [015001]. https://doi.org/10.1088/1681-7575/ac36b6

\section{General rights}

Copyright and moral rights for the publications made accessible in the public portal are retained by the authors and/or other copyright owners and it is a condition of accessing publications that users recognise and abide by the legal requirements associated with these rights.

- Users may download and print one copy of any publication from the public portal for the purpose of private study or research.

- You may not further distribute the material or use it for any profit-making activity or commercial gain

- You may freely distribute the URL identifying the publication in the public portal 
PAPER • OPEN ACCESS

\section{Prenormative verification and validation of a} protocol for measuring magnetite-maghemite ratios in magnetic nanoparticles

To cite this article: Lara K Bogart et al 2022 Metrologia 59015001

View the article online for updates and enhancements.
You may also like

\begin{tabular}{l} 
- Physicochemical Properties of Nonvariant \\
$\frac{\text { Compositions of Ternary NaF-LiF-LnF }}{3}$ \\
$\frac{\text { Systems (Ln }=\text { La, Nd) }}{\text { Nataliya V Faidyuk, Ruslan N Savchuk }}$ \\
and Anatolii A Omel'chuk \\
- Development of Long Life and Low Cost \\
$\underline{\text { Li-Rich Layered Oxide Positive Active }}$ \\
Material for xEVs \\
Katsuya Inoue, Shigeki Yamate, Ji-Yong \\
Shin et al. \\
- Electrical Properties of $\mathrm{Al}_{2} \mathrm{O}_{3}$ Incorporated \\
\hline CeO Thin Films Deposited by RF \\
Magnetron Sputtering \\
Junya Konishi, Takashi Ohsawa, Setsu \\
Suzuki et al.
\end{tabular}




\title{
Prenormative verification and validation of a protocol for measuring magnetite-maghemite ratios in magnetic nanoparticles
}

\author{
Lara K Bogart ${ }^{1}\left(\mathbb{D}\right.$, Jeppe Fock ${ }^{2,3}{ }^{\oplus}$, Geraldo M da Costa ${ }^{4}$, Kerstin Witte $^{5,6}$, \\ Jean-Marc Greneche ${ }^{7}{ }^{(}$, Jan Zukrowski ${ }^{8}$, Marcin Sikora ${ }^{8}{ }^{\circ}$, Drew E Latta ${ }^{9}$, \\ Michelle M Scherer ${ }^{9}$, Mikkel Fougt Hansen ${ }^{2}{ }^{\circ}$, Cathrine Frandsen ${ }^{10} \odot$ and \\ Quentin A Pankhurst ${ }^{1, *}$ (1)
}

${ }^{1}$ Healthcare Biomagnetics Laboratory, University College London, 21 Albemarle Street, London W1S 4BS, United Kingdom

2 DTU Health Tech, Technical University of Denmark, DK-2800 Kongens Lyngby, Denmark

3 Blusense Diagnostics APS, DK-2100 Copenhagen E., Denmark

${ }^{4}$ Departamento de Quimica, Universidade Federal de Ouro Preto, Ouro Preto, MG, Brazil

5 Institut für Physik, Universität Rostock, Albert-Einstein-Straße 23, 18059 Rostock, Germany

${ }^{6}$ INP Leibniz-Institut für Plasmaforschung und Technologie, Felix-Hausdorff-Straße 2, 17489 Greifswald, Germany

${ }^{7}$ Institut des Molécules et Matériaux du Mans, IMMM UMR CNRS 6283 Le Mans Université, 72085 Le Mans Cedex 9, France

8 Academic Centre for Materials and Nanotechnology, AGH University of Science and Technology, 30-059 Krakow, Poland

${ }^{9}$ Civil and Environmental Engineering, University of Iowa, Iowa City, Iowa 52240, United States of America

${ }^{10}$ DTU Physics, Technical University of Denmark, DK-2800 Kongens Lyngby, Denmark

E-mail: q.pankhurst@ucl.ac.uk

Received 24 October 2021

Accepted for publication 4 November 2021

Published 3 December 2021

\begin{abstract}
An important step in establishing any new metrological method is a prenormative interlaboratory study, designed to verify and validate the method against its stated aims. Here, the ${ }^{57} \mathrm{Fe}$ Mössbauer spectrometric 'centre of gravity' (COG) method was tested as a means of quantifying the magnetite/maghemite $\left(\mathrm{Fe}_{3} \mathrm{O}_{4} / \gamma-\mathrm{Fe}_{2} \mathrm{O}_{3}\right)$ composition ratio in biphasic magnetic nanoparticles. The study involved seven laboratories across Europe and North and South America, and six samples - a verification set of three microcrystalline mixtures of known composition, and a validation set of three nanoparticle samples of unknown composition. The spectra were analysed by each participant using in-house fitting packages, and ex post facto by a single operator using an independent package. Repeatability analysis was performed using Mandel's $h$ statistic and modified Youden plots. It is shown that almost all $(83 / 84)$ of the Mandel $h$ statistic values fall within the $0.5 \%$ significance level, with the one exception being borderline. Youden-based pairwise analysis indicates the dominance of
\end{abstract}

* Author to whom any correspondence should be addressed 
random uncertainties; and in almost all cases the data analysis phase is only a minor contributor to the overall measurement uncertainty. It is concluded that the COG method is a robust and promising candidate for its intended purpose.

Keywords: magnetic nanoparticles, ${ }^{57} \mathrm{Fe}$ Mössbauer spectrometry, iron oxide, magnetite/maghemite

S Supplementary material for this article is available online

\section{Introduction}

Magnetic nanoparticles comprising mixtures of the iron oxides magnetite $\left(\mathrm{Fe}_{3} \mathrm{O}_{4}\right)$ and maghemite $\left(\gamma-\mathrm{Fe}_{2} \mathrm{O}_{3}\right)$ have been the subject of sustained and significant R \& D interest for many years, with ca 25000 publications to date, including ca 200 per month in the last 5 years [1]. To a large extent, this interest is driven by their utilisation in biomedical applications, where their inherent biocompatibility and safety profile is well established, and their intrinsic magnetic properties lead to a variety of mechanisms of action, from sensing and imaging, and actuation and targeting, through to hyperthermia [2-5].

The biological environment is also the one in which the magnetite/maghemite characterisation of these materials is most pertinent, given an increasing understanding of the importance of their redox-active character, stemming from the presence of $\mathrm{Fe}^{2+}$ ions as well as $\mathrm{Fe}^{3+}$ ions in magnetite, as opposed to $\mathrm{Fe}^{3+}$-only ions in maghemite. For example, potentially beneficial actions such as macrophage phenotype activation [6, 7] and enzyme mimetics [8] have been reported, while at the same time concerns are expressed about the uncontrolled production of reactive oxygen species via the Haber-Weiss and Fenton reactions $[9,10]$.

However, almost all of these biological applications rely specifically on the nanoscale form, where the absence of remanent magnetisation-due to the size-dependent phenomenon of superparamagnetism [2] - removes the risk of agglomeration and embolism in vivo. This is beneficial for their intended use, but it makes their characterisation much more difficult than it is for bulk materials. This is because, at the nanoscale, experimental effects such as the broadening of $\mathrm{x}$-ray diffraction lines make distinguishing magnetite from maghemite virtually impossible. The bulk magnetisations of magnetite and maghemite differ by only a few percent, so that distinguishing them magnetically is difficult even in single-phase samples, let alone in mixtures, where the component masses are themselves hard to assess. This is a longstanding problem in the field, which has led to a tendency for researchers to report their findings with reference to an unspecified 'magnetite/maghemite' material, rather than attempt to interrogate the actual composition.

There is, however, one technique that is especially well suited for quantifying the magnetite and/or maghemite content in magnetic nanoparticles, viz ${ }^{57} \mathrm{Fe}$ Mössbauer spectrometry [11-16]. Although not as routinely available as, e.g., X-ray diffraction or bulk magnetometry, ${ }^{57} \mathrm{Fe}$ Mössbauer spectrometry is widely practised, and facilities are found in most countries [17]. It is a form of nuclear gamma-ray spectroscopy that has the advantage of directly interrogating the immediate atomic environment of the ${ }^{57} \mathrm{Fe}$ isotope atoms within a sample. As such it is especially well suited to distinguishing between $\mathrm{Fe}^{2+}$ and $\mathrm{Fe}^{3+}$ ions in the solid state.

In the last decade, a measurement protocol known as the 'centre of gravity' (COG) method has been developed that uses ${ }^{57} \mathrm{Fe}$ Mössbauer spectrometry to measure the magnetite/maghemite ratio in iron-oxide-based magnetic nanoparticles [11-16]. In brief: according to the COG method, a single parameter, $\bar{\delta}_{\mathrm{RT}}$ - the 'COG', or area weighted mean isomer shift at room temperature - is extracted by curve-fitting a spectrum using a partly-constrained superposition of Lorentzian singlets, doublets, and sextets [18]. In the context of the COG method, the $\bar{\delta}_{\mathrm{RT}}$ parameter correlates with the number of $\mathrm{Fe}$ atoms in the magnetite environment:

$$
\alpha=\left[\mathrm{Fe}_{\text {magnetite }}\right] /\left[\mathrm{Fe}_{\text {total }}\right]=\left(\bar{\delta}_{\mathrm{RT}}-\delta_{\mathrm{o}}\right) / m,
$$

where the constants $\delta_{\mathrm{o}}=0.3206 \mathrm{~mm} \mathrm{~s}^{-1} \pm 0.0022 \mathrm{~mm} \mathrm{~s}^{-1}$ and $m=0.2135 \mathrm{~mm} \mathrm{~s}^{-1} \pm 0.0076 \mathrm{~mm} \mathrm{~s}^{-1}$ have been experimentally determined using calibrated samples [15].

Equation (1) contains two parts, which are important to disentangle for the analysis that follows. The first part, $\alpha=\left[\mathrm{Fe}_{\text {magnetite }}\right] /\left[\mathrm{Fe}_{\text {total }}\right]$, is an expression of the heart of the COG hypothesis: viz that $\alpha$ correlates with the atomic percentage of $\mathrm{Fe}$ atoms present in the form of magnetite, relative to the total number of $\mathrm{Fe}$ atoms in the material. It a strict sense, this part is interpretative. It is applicable only when magnetite and maghemite are the only Fe-containing phases present, and only when the $\alpha$ parameter lies in the range from 0 to 1 .

The second part, $\alpha=\left(\bar{\delta}_{\mathrm{RT}}-\delta_{\mathrm{o}}\right) / m$, is the mathematical definition of the metric. Given that $\bar{\delta}_{\mathrm{RT}}$, as the area weighted mean isomer shift of a spectrum, can in principle take any value, and given that both $\delta_{\mathrm{o}}$ and $m$ are constants, it is clear that mathematically, $\alpha$ is an unbounded parameter. As such, one should anticipate a Gaussian sampling distribution in measurements of $\alpha$. Furthermore, one should anticipate that even with a material comprising solely magnetitie and/or maghemite, it will be possible to measure $\alpha$ values that may be less than zero or greater than one.

Returning to the interpretative aspect of equation (1), one of the reasons this metric has attracted attention is that it makes accessible and quantitative a material characteristic that is otherwise very difficult to measure [19]. For example, the $\alpha$ 
parameter may be related to more discipline-specific metrics [15], such as the molar ratio of ferrous and ferric ions:

$$
x=\mathrm{Fe}^{2+} / \mathrm{Fe}^{3+}=\alpha /(3-\alpha),
$$

which is commonly used in chemical characterisation; or the weight percentage of the magnetite phase:

$$
w=M_{\text {magnetite }} / M_{\text {total }}=28.94 \alpha /(29.94-\alpha)
$$

in the mixture, which is commonly use in mineralogy.

In light of this, our aspiration is to support the transition of the COG method from its current prenormative level to that of a normalised, ISO standard method for the quantification of the magnetite/maghemite ratio in magnetic nanoparticles. We believe that such an ISO standard is needed to support the companies and industries that trade in these high-technology, high-value materials, especially when they are used in the biomedical sector, either as medical devices or as components of medicinal products.

Such an effort is timely. Recent work on the metrology of magnetic nanoparticles has led to the formulation of a standardisation roadmap [20] and the introduction of two new definition standards, ISO 19807-1 and ISO 19807-2 [21]. Measurement standards have not yet been prepared, but they are a logical next step. For the COG method in particular, one of the key underpinning requirements of a new measurement standard - that of having in place a well-defined uncertainty budget for the measurand-has recently been published [14]. Another metrological requirement - that of having traceability to SI units - is entirely achievable [22], even if it has not yet been formally presented. It is anticipated that establishing the traceability chain will be straightforward, should the opportunity arise to propose the COG method as a measurement standard.

In parallel, efforts have been made to explore better, more robust characterisation methods for magnetic nanoparticles-including the COG method-via consortia of laboratories interested in advancing the field [25]. This is advantageous, as the method of choice for prenormative verification and validation of a newly proposed measurement protocol is interlaboratory comparison [24].

We therefore report here on an international project in which seven groups tested the COG method by recording and analysing ${ }^{57} \mathrm{Fe}$ Mössbauer data from six iron oxide samples-a verification set of three microcrystalline mixtures of predetermined composition, and a validation set of three nanoparticulate samples of a priori unknown composition. With reference to the American Society for Testing and Materials (ASTM) standard practice guide for interlaboratory comparisons of test methods [24], we report on the statistical analysis of both datasets using both Mandel- and Youdenstyle repeatability tests. Based on this interlaboratory comparison, we conclude that the COG protocol is applicable with reasonable uncertainty and bias, and that as such the results as presented could serve as the basis for development of a new normalisation standard for the quantification of the magnetite/maghemite ratio in magnetic nanoparticles.

\section{Experimental methods}

Study design: experienced practitioners from seven laboratories-five European, one North American, and one South American-participated in the study. The study was coordinated by the University College London (UCL) and Technical University of Denmark (DTU) teams, who selected and prepared six samples, from each of which seven identical aliquots were extracted [section S1 (https://stacks.iop. org/MET/59/015001/mmedia)]. One aliquot from each sample was delivered to the study participants in the form of undiluted, free-flowing powders, in sufficient quantities to allow suitable-thickness absorbers to be prepared. The samples were anonymised, and no information as to the composition or nature of the samples was divulged. All participants were asked to prepare absorbers suitable for their experimental conditions, and to collect a room temperature spectrum for each sample (section S2). Next, the participants were asked to analyse their spectra according to their usual in-house procedures (including in-house operators and analysis programs) but following published instructions regarding the COG method [15]; and to return both the COG analysis results as well as a complete set of all raw data, including calibration files, to both UCL and DTU. At UCL the data thus collated was anonymised prior to repeatability analysis, which comprised two parts. Part 1 was a statistical analysis of the as-received COG analysis results ( $\alpha$ values) of each of the six samples; and part 2 was a variant of part 1 in which the raw data files from each of the participants were independently analysed by a single operator (one of the authors, JF, using a single data analysis program) to extract a second set of COG $\alpha$ values for analysis.

Samples and absorber preparation: the samples included both verification and validation sets. The verification samples (NM-A, NM-B and NM-C) were microcrystalline powder mixtures prepared at DTU using well-characterised maghemite and magnetite powders (section S1) [15]; and predetermined $\alpha$ values of $0.109 \pm 0.002,0.683 \pm 0.014$ and $0.97 \pm 0.02$ respectively (table $\mathrm{S} 1$ ). The validation samples were all superparamagnetic nanoparticles - meaning that they had zero net magnetisation at room temperature-and had a priori unknown $\alpha$ values. Two (NM-D and NM-E) were manufactured by Micromod GmbH (Rostock, Germany) [25]; while the third, NM-F, was a powder sample taken 'from the shelf' at DTU, which, according to the logbook, was synthesized by a route similar to that for uncoated, freeze-dried maghemite nanoparticles from that laboratory [26]. To guard against oxidative ageing effects, which are known to be most significant in the first few weeks and months after synthesis [16], all three validation samples were at least 16 months old before measurements were performed (tables S6 and S9). Absorber preparation methods - including the choice of sample holder and absorber area, the mixing of the samples with a $\gamma$-ray transparent dispersant medium such as sucrose or boron nitride to facilitate texture-free packing, and their mounting in an Fe-free absorber holder - were left to the discretion of each participant (table S2). However, all participants were asked to 
limit the maximum spectral absorption to $8 \%$, and to aim for a value from $6 \%$ to $8 \%$.

Measurements and data analysis: no specifications were given regarding the choice of velocity (energy) scale, other than for each spectrum to have a flat background. Participants were asked that their spectrometer hardware should be free from vibrations and that the collected spectra should exhibit what they considered to be qualitatively 'good' linewidths, 'good' alignment and 'good' quality statistics (table S3). Participants were asked to measure each sample at room temperature (and to record that temperature), and to calibrate their spectrum relative to an $\alpha$-Fe foil. They were asked to then analyse their spectra using their usual fitting programs, but to apply the COG method to obtain the area weighted mean isomer shift $\left(\bar{\delta}_{\mathrm{RT}}\right)$ and the corresponding $\alpha$ parameter determined from equation (1). Participants were also advised that, if available in their laboratories and if they considered it appropriate, additional measurements could be made at lower-than-room temperature using a cryostat or similar controlled environment. (This was because low temperatures could be expected to reduce linewidths, and improve the determination of $\alpha$.) In such cases, participants were asked to report both their measured $\bar{\delta}(T)$ and the measurement temperature, and their derived values for $\alpha$ using a previously published correction formula (equation 17 in [15]). Lastly, participants were also asked to provide copies of their as-collected raw data (sample and $\alpha$-Fe calibration files) for consolidation at UCL. Following this, all of the spectra were re-analysed by a single operator at DTU using a custom-built Matlab script.

Uncertainty budget: in evaluating the $\bar{\delta}_{\mathrm{RT}}$ values from their acquired data, no constraint was imposed on the participants, and they were simply asked to apply their in-house protocols. However, for the single-operator re-analyses, the uncertainty budgets of each data point was independently evaluated in line with published protocols [14]. These comprised: uncertainties associated with the fitting of the Mössbauer spectra; calibration of the $\alpha$-Fe reference spectrum; post facto treatment of the data (viz taking account of the temperature-dependent second order Doppler shift, for absorber temperatures above or below $295 \mathrm{~K}$ ); and experimental errors (viz testing for deviations from a flat background or for non-linear velocity-channel calibrations) [14].

Repeatability analysis: to analyse repeatability, the approaches described by the American Society for Testing and Materials in their ASTM E691-18 report on 'Standard Practice for Conducting an Interlaboratory Study to Determine the Precision of a Test Method' [24] were applied.

\section{Results}

\subsection{Overview of measured spectra}

Figure 1 shows the full set of recorded data from one of the participating laboratories, laboratory 6 .

The spectra of the verification samples, from the magnetiterich NM-A to the almost-pure maghemite NM-C, all have sharp absorption line profiles, as is usual in microcrystalline

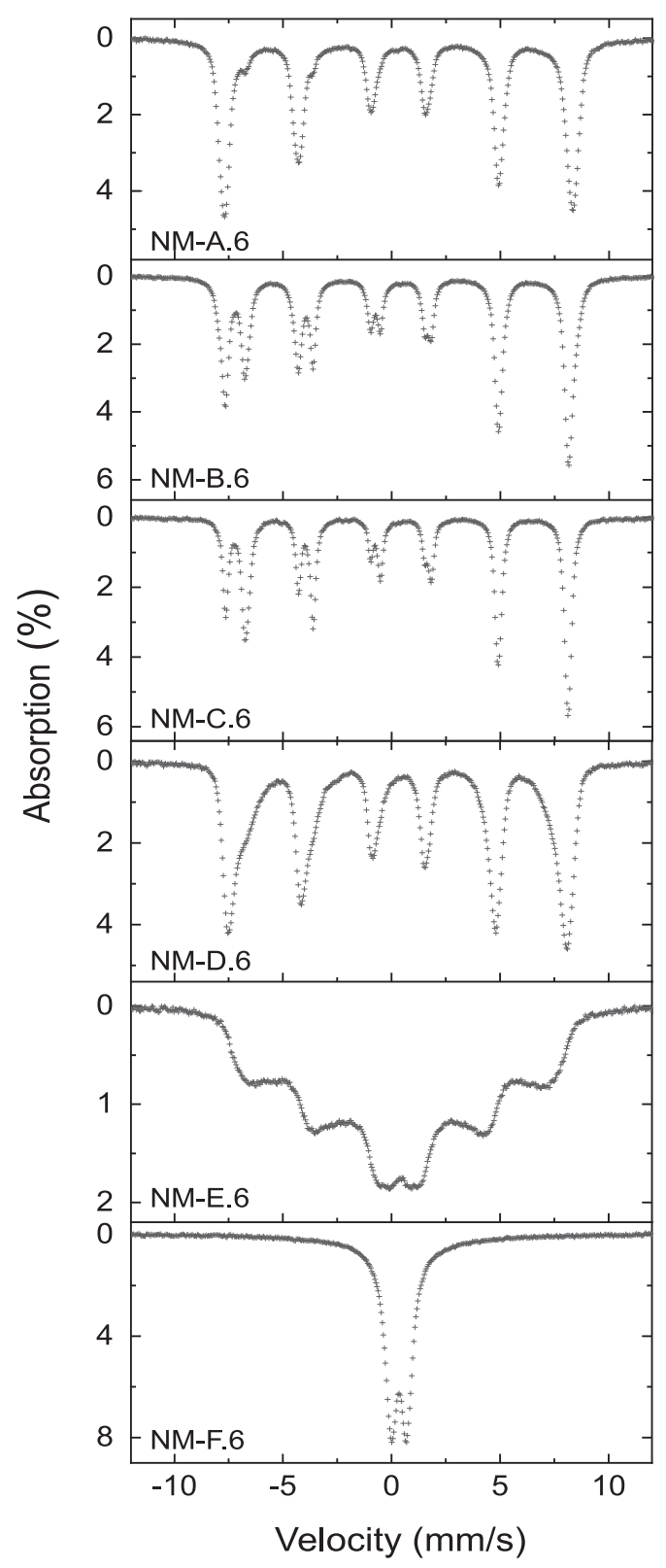

Figure 1. ${ }^{57} \mathrm{Fe}$ Mössbauer spectra, recorded at room temperature $(T=295 \mathrm{~K} \pm 5 \mathrm{~K})$, of six representative iron oxide samples chosen for the interlaboratory study, viz three microcrystalline mixtures of magnetite and maghemite (NM-A to -C) and three nanoparticulate iron oxide samples (NM-D to -F).

mixtures. In contrast, the nanoparticulate samples in the validation set exhibit much more varied profiles, ranging from an asymmetrically broadened sextet (NM-D), through a spectrum resembling a 'terraced valley' (NM-E), to a relatively sharp doublet with broad 'wings' (NM-F).

It may be noted in passing that although all three of the nanoparticulate samples were superparamagnetic, they did not all exhibit doublet spectra, and indeed two exhibited the sextet features typical of magnetically ordered states. This may seem incongruous, but in fact it is a common feature of the ${ }^{57} \mathrm{Fe}$ Mössbauer spectrometry of nanomaterials, namely that 

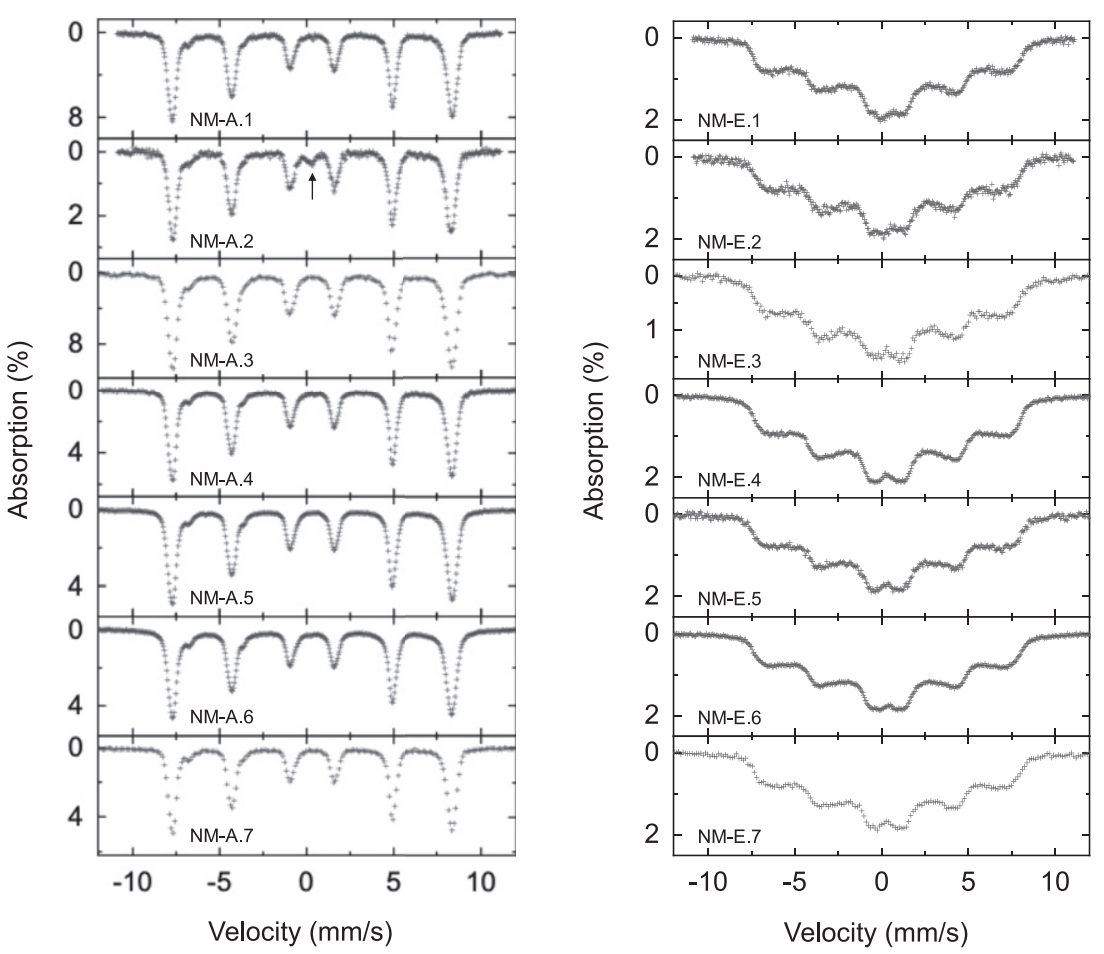

Figure 2. (a) Comparison of the as-received room temperature ${ }^{57} \mathrm{Fe}$ Mössbauer spectra recorded in seven different laboratories for a microcrystalline magnetite-maghemite mixture, sample NM-A. An arrow indicates the presence of an anomalous additional absorption line, hypothesized as being due to impurities in the sample holder, in spectrum NM-A.2. (b) Comparison of the as-received room temperature ${ }^{57} \mathrm{Fe}$ Mössbauer spectra recorded in seven different laboratories for a nanoparticulate magnetite-maghemite, sample NM-E.

on the relatively short measurement timescale of the technique-which is determined by the Larmor precession frequency of the ${ }^{57} \mathrm{Fe}$ nucleus and is of order nanoseconds- the nuclear hyperfine fields may well be time-invariant, while at the longer timescale of, e.g., bulk hysteresis measurements, the net magnetisation averages to zero [12].

The raw data received from each of the laboratories are shown in figure 2(a) for one of the microcrystalline mixtures (NM-A) and in figure 2(b) for one of the nanomaterials (NM-E), and in figures S1 and S2 for the remaining samples.

At first glance, all the spectra appear to be very similar, however, on closer inspection some differences are apparent. First, an extraneous absorption line at velocity $v \approx 0.3 \mathrm{~mm} \mathrm{~s}^{-1}$ is visible in NM-A.2. On investigation, a likely explanation for this was found to be that laboratory 2 used non-certified aluminium foil as a sample holder, which, strictly speaking, is not best practice, as it is known that Fe impurities in aluminium present Mössbauer spectra with singlets in the velocity range from 0 to $0.4 \mathrm{~mm} \mathrm{~s}^{-1}$ [27].

Second, the absorption percentages vary, which raises a question as to 'thickness effects' due to self-absorption in the absorber [28], which may lead to analysis artefacts [29]. However, on investigation this was found not to be the case. Rather, the absorption differences were found to be due to the variance in source-sample-detector geometries in the different laboratories. This technical feature affects the non-resonant background levels in the spectra, and accounts for the observed absorption variations.
Third, there are differences in the extent to which the measured baseline extends beyond the absorption region, which might affect the accuracy of the baseline determination which forms part of the data analysis and be reflected in larger uncertainties. That said, all the laboratories used the current bestpractice of a triangular drive waveform and folded their data to give a spectrum with a hypothetically flat baseline.

Fourth, there is variation in the statistical quality of the measured data, as evidenced by the scatter of the individual channel counts in the spectra. As well as affecting the random-uncertainty-based uncertainties associated with each measurement, a lack of sufficient statistical quality may lead to a lack of definition in the fit. At the same time, over-long data acquisition is also problematic, as excess statistical quality can lead to difficulties in data analysis with poorly defined sum-of-least-squares minima.

On the basis of these observations as to the quality of the recorded data, it was determined that all were representative of current norms in ${ }^{57} \mathrm{Fe}$ Mössbauer spectroscopy, and as such they constituted an appropriate sampling for the COG method test.

\subsection{Fitted $\alpha$ values}

The $\alpha$ values obtained from each laboratory-as determined both in-house using each participants' usual spectrum fitting packages (tables S4 and S7), and ex post facto by a single operator using an independent analysis package (tables S5, 

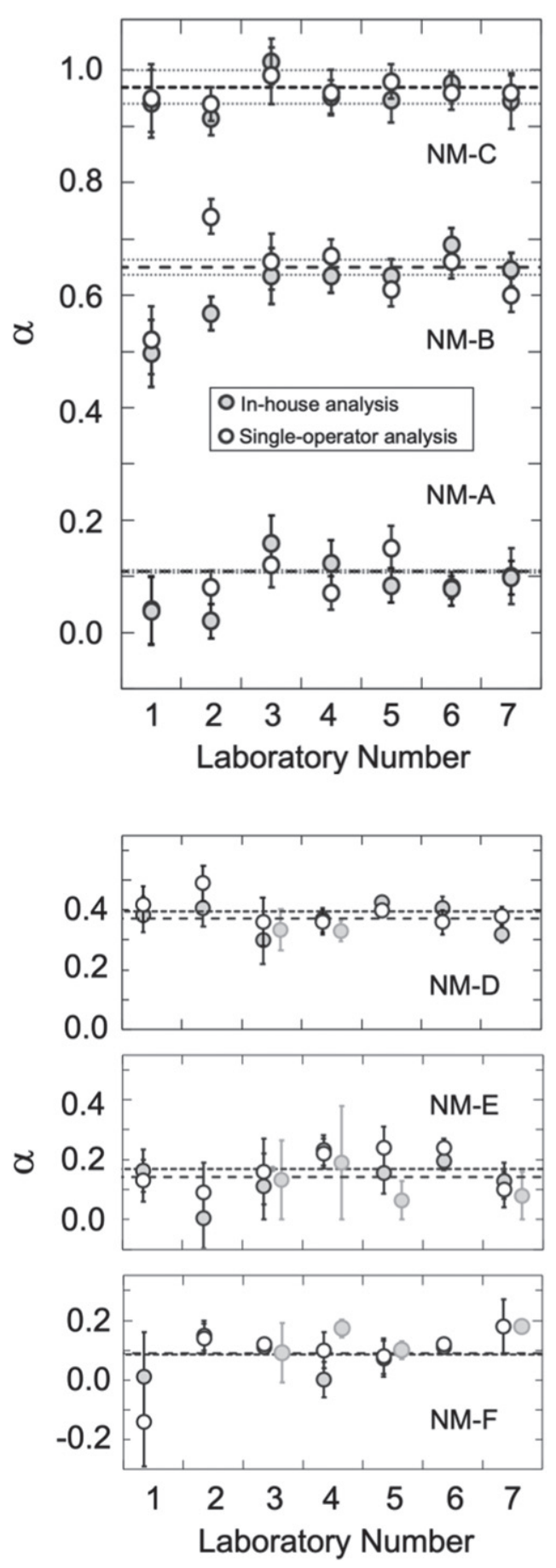

Figure 3. (a) Measured $\alpha$ values derived from room temperature Mössbauer spectra for the microcrystalline mixtures NM-A to -C, from both in-house and single-operator analysis. The superimposed dashed and dotted lines represent the a priori known $\alpha$ values and their uncertainties. (b) Measured $\alpha$ values derived from room temperature Mössbauer spectra for the nanoparticulate iron oxides NM-D to -F, from both in-house and single-operator analysis. The dashed and dotted lines represent the calculated means of the $\alpha$ values using in-house and single-operator room temperature data respectively. Some additional data points (grey outline) were obtained from spectra recorded at liquid nitrogen temperature or, for spectrum NM-E.5, at $T=140 \mathrm{~K} \pm 2 \mathrm{~K}$.
Table 1. Averaged data from 7 independent Mössbauer laboratories' measurements of the COG method $\alpha$ values of 6 different iron oxide samples, from both in-house and single-operator analysis. The uncertainties listed are the standard deviations of the measurements.

\begin{tabular}{lccc}
\hline Sample & Expected & In-house & Single-operator \\
\hline NM-A & $0.109 \pm 0.002$ & $0.09 \pm 0.05$ & $0.09 \pm 0.04$ \\
NM-B & $0.683 \pm 0.014$ & $0.61 \pm 0.06$ & $0.64 \pm 0.07$ \\
NM-C & $0.97 \pm 0.02$ & $0.96 \pm 0.03$ & $0.96 \pm 0.02$ \\
NM-D & - & $0.37 \pm 0.05$ & $0.40 \pm 0.05$ \\
NM-E & - & $0.14 \pm 0.07$ & $0.17 \pm 0.06$ \\
NM-F & - & $0.09 \pm 0.07$ & $0.09 \pm 0.10$ \\
\hline
\end{tabular}

S8, and S10) - are shown in figures 3(a) and (b) and compared therein to the known true $\alpha$ values (for the mixtures) or to the measured mean $\alpha$ values (for the nanoparticles). Also shown are the $\alpha$ values determined by four laboratories $(3,4,5$, and 7$)$ that recorded spectra of the nanoparticle samples at reduced temperatures (see figure S3 for further details of these measurements). In keeping with the unbounded nature of the $\alpha$ parameter-which was discussed in section 1 in relation to the mathematical form of equation (1)-it may be noted that in some cases the measured $\alpha$ values, and/or their standard deviation limits, fall outside the range from zero to one.

The averaged data across all 7 laboratories are listed in table 1. On collation of the in-house data, it was noted that laboratories 1, 2, and 3 had used Lorentzian line profiles rather than the suggested Voigtian line profiles in their fits (a Voigtian being a Gaussian distribution sum of Lorentzian lines [30]). It was decided to leave this unchanged, but Voigtian profiles were used for all the single-operator re-analysis fits. Generally good agreement is seen in the in-house data, with the possible exception of the data for NM-A and -B from laboratories 1 and 2. The single-operator analysis affected the fitted $\alpha$ values slightly, but always within the uncertainty of the in-house value, and with no clear trend between the in-house and singleoperator values. Data from the low temperature experiments, where performed, was comparable with the room temperature data.

\subsection{Repeatability analysis}

Following ASTM guidance [24], two well-known repeatability analysis methods were applied to the data, viz Mandel's consistency statistic [24] and the Youden plot [31]. The goal here was to identify outliers and to gain insight into the random and systematic uncertainties, and the statistical confidence, in the measurements.

3.3.1. Mandel's consistency statistic. The $h$ and $k$ consistency metrics developed by Mandel are ways to assess the repeatability and reproducibility in interlaboratory studies $[32,33]$. As the participants measured only one spectrum per 
sample, the $k$ statistic was not valid, and was not considered further, and the analysis focused instead on the $h$ statistic.

Briefly, the $h$ statistic is the deviation of each measurement from the overall average of the measured values, divided by the standard deviation of those measured values. It is mathematically equivalent to Grubb's outlier statistics [33], and is given here by:

$$
h=(\alpha-\bar{\alpha}) / s_{x},
$$

where $\bar{\alpha}$ is the mean $\alpha$ value for each sample and $s_{x}$ is the standard deviation of all of the individual measurements from each laboratory. In this way, $h$ is a measure of the deviation of a given measurement from the nominal value, and a way to identify outliers, as well as being a statistic for a test of significance. For a given significance level, say $2 x \%$, a critical value of $h$ may be determined from:

$$
h_{\text {crit }}=(p-1) t / \sqrt{p\left(t^{2}+p-2\right)},
$$

where $p$ is the number of independent measurements, and $t$ is the value of the upper $(1-x)^{\text {th }}$ percentile of the student's $t$ distribution with $(p-2)$ degrees of freedom [24]. Although this may look complicated, it is easily calculated using readily available spreadsheet functions: e.g. at the $0.5 \%$ significance level $(x=0.25 \%=0.0025$, with $t$ being the value of the upper $99.75^{\text {th }}$ percentile), $t$ is given in Microsoft Excel by TINV $(0.005, p-2)$, where TINV is the student's $t$ inverse cumulative distribution function.

The $h$ statistics for each of the samples for all 7 laboratories are shown in figures 4(a) and (b). As before, data is shown for the $\alpha$ values determined by the individual participants, and by the single-operator analysis. For both the mixtures and the nanoparticles, different $\bar{\alpha}$ values were used for the in-house and single-operator datasets: in each case $\bar{\alpha}$ was taken to be the mean of the measured $\alpha$ values from all 7 laboratories, as per the respective analysis. Also shown in figure 4, following the ASTM guidance, [24] are dashed lines at the $h_{\text {crit }}= \pm 2.05$ values that correspond to the $0.5 \%$ significance level for $p=7$ independent measurements, calculated using equation (5).

For the mixtures (in-house analysis), it is apparent that most $(15 / 21)$ of the measured $h$ statistics lie within \pm 1 standard deviation $s_{x}$ of the true values; while the remainder $(6 / 21)$ lie in the range from $\pm 1 s_{x}$ to $\pm 2 s_{x}$. None lie outside the $0.5 \% t$ test significance level. Single-operator analysis of the same data modifies the spread of data slightly, with 14/21 data points now within $\pm 1 s_{x}, 7 / 21$ from $\pm 1 s_{x}$ to $\pm 2 s_{x}$, and again none beyond the $0.5 \%$ level. More generally, figure 4 shows that the single-operator analysis affected the individual $h$ statistics, but without any clearly discernible trend: in some cases, the magnitude of $h$ increased, and in some cases it decreased.

For the nanoparticles (in-house analysis), most (13/21) of the measured $h$ statistics lie within $\pm 1 s_{x}$ of the respective mean values; and the remainder (8/21) lie in the range from $\pm 1 s_{x}$ to $\pm 2 s_{x}$. Single operator analysis again slightly changes the spread, with $15 / 21$ data points now within $\pm 1 s_{x}, 5 / 21$ from $\pm 1 s_{x}$ to $\pm 2 s_{x}$, and $1 / 21$ just beyond the $0.5 \%$ level. As with the mixtures, no clear trend towards either larger or smaller $h$ statistics is apparent in the single-operator analysis data.
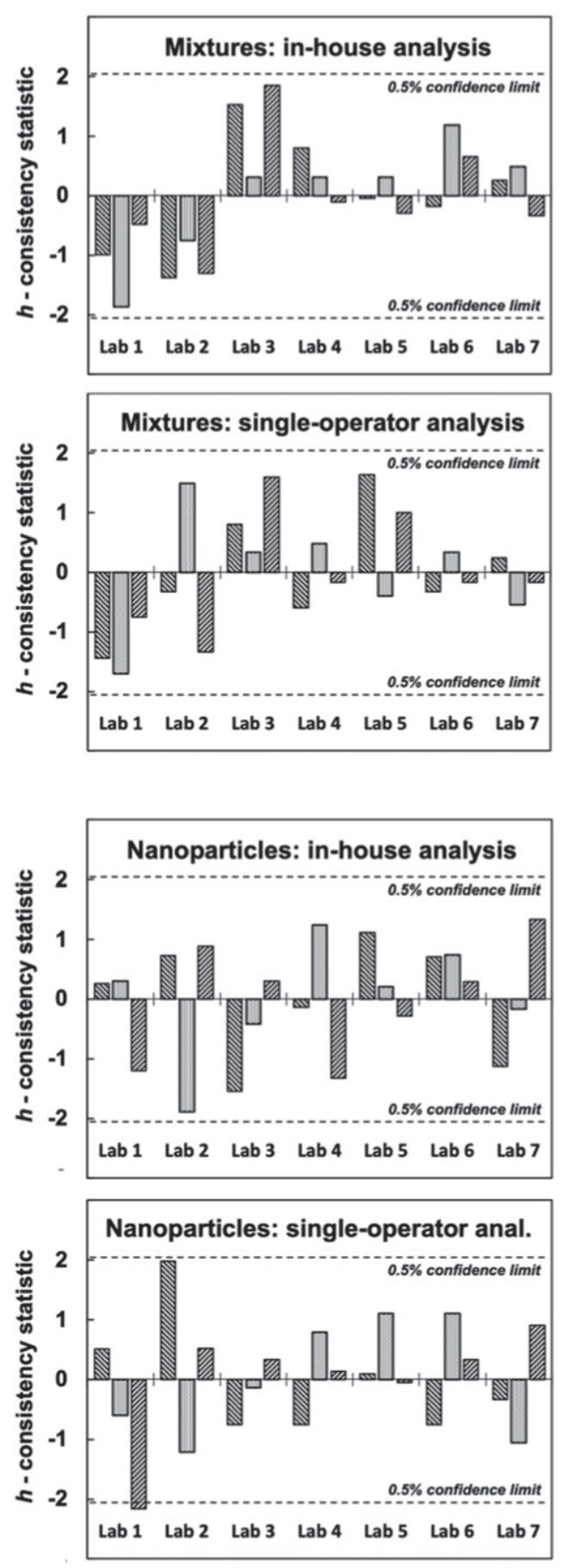

Figure 4. (a) Values of Mandel's $h$ statistic, measured against the known values of $\alpha$ for each of the mixtures NM-A to $-\mathrm{C}$, for both in-house and single-operator data analysis. The dashed lines indicate $t$ test significance levels corresponding to the $0.5 \%$ significance level for $N=7$ independent measurements. (b) Values of Mandel's $h$ statistic, measured against the average measured value of $\alpha$ for each of the nanoparticles NM-D to -F, for both in-house and single-operator data analysis. The dashed lines indicate $t$ test significance levels corresponding to the $0.5 \%$ significance level for $N=7$ independent measurements.

3.3.2. Youden analysis. The Youden plot is a means of uncovering bias in a measurement process, and is designed to identify and distinguish between random and systematic 

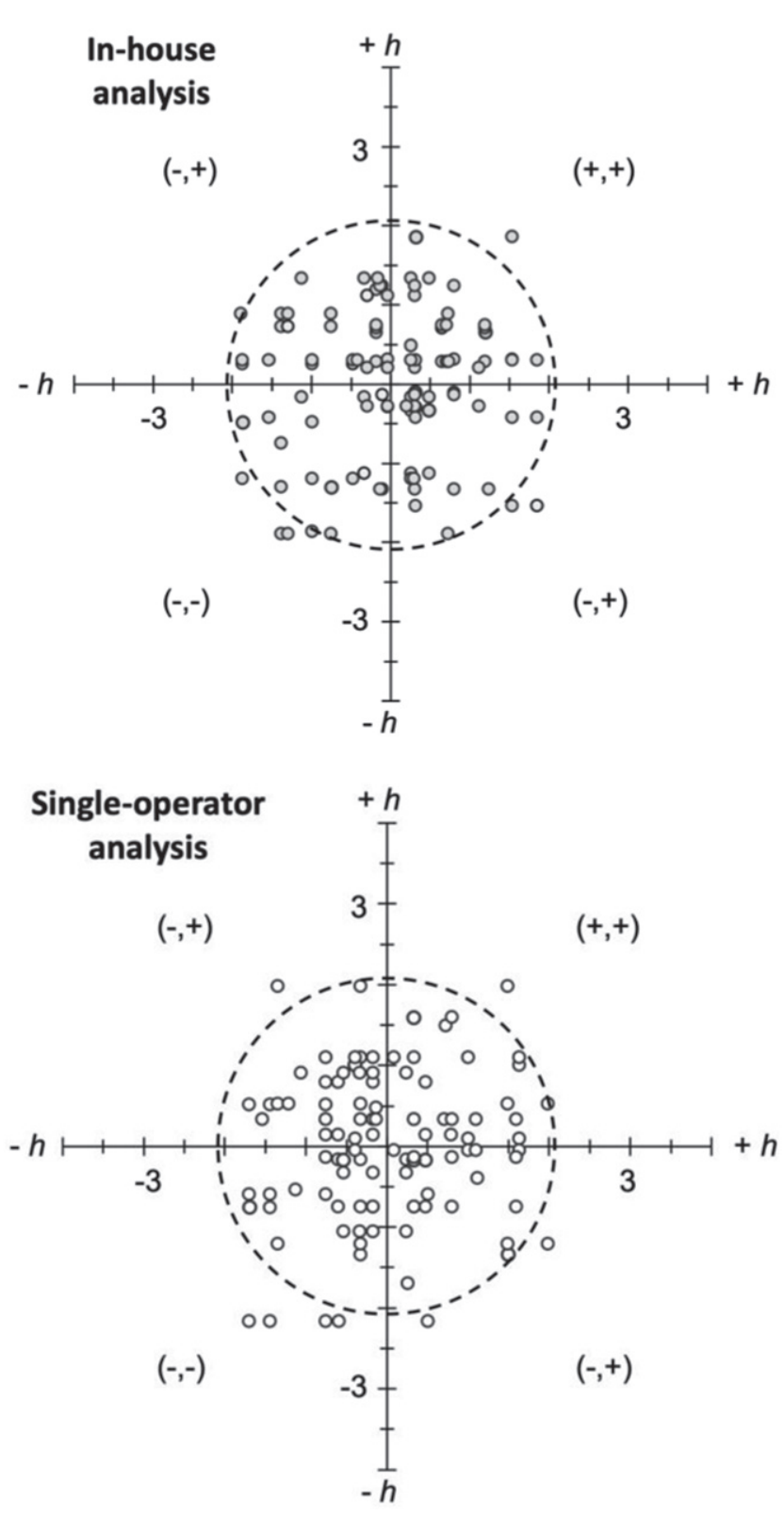

Figure 5. Youden-style pairwise plots of the Mandel $h$ statistic (in units of standard deviation $s_{x}$ ) derived from the Mössbauer effect $\alpha$ values measured, in seven laboratories, for three microcrystalline and three nanoparticulate samples, via in-house and single-operator analysis. The dashed lines mark out circles at the $0.5 \% t$ test significance level for $N=7$ independent measurements. The absence of any clear deviation in the clusters towards elliptical symmetry indicates that systematic uncertainty effects, if present, are small compared to random uncertainty effects.

uncertainties [31]. It is usually applied when two or more laboratories make measurements on two similar samples that are close in the magnitude of the property (parameter) evaluated. This was not the case in the current study. Nevertheless, a modified Youden plot was here considered useful, wherein pairwise comparisons were made between samples-viz NM-A versus -B; NM-A versus -C, NM-B versus -C, etc-and wherein the Mandel $h$ statistics were plotted rather than the measured $\alpha$ values. Although unconventional, computer simulations of the distribution of $h$ statistic pairs, in the case of a Gaussian sampling distribution, indicated that the approach was robust (see figure S4). As such, it is the authors' opinion that this was an acceptable way to visualise the data without the complications that arise when the measured properties in the twosample plots are of different magnitude. It was further decided to plot the pairwise data from the full set of samples - mixtures and nanoparticles both-on the basis that this would allow the greatest number of pairwise comparisons to be made (viz 105 data points for each of the in-house and singleoperator sets).

The resulting Youden-style plots are shown in figure 5. (More detailed versions, including individual labels for each of the data points, are given in figures S5 and S6.) To interpret this data, it is useful to note that in a Youden plot, if there are only random uncertainties, all the points are expected to cluster about the origin and lie within a circle. If there is a predominance of systematic uncertainty over random uncertainty, the encompassing circle is expected to elongate along the $45^{\circ} / 225^{\circ}$ axis (i.e. the line bisecting the first and third quadrants) to form an ellipse. In such a case, most of the data points would be expected to fall within the first and third quadrants - marked $(+,+)$ and $(-,-)$ respectively in figure 5 .

Inspection thus shows that for both the in-house analysis and the single-operator analysis, 97/105 points lie inside the circle of radius corresponding to the $0.5 \% t$ test significance level, indicating good repeatability in both cases. There is also no discernible bias in the data-the data points appear to be evenly spread over the plane, and there is no evidence of a significant preference for the first and third quadrants. As such, the data are consistent with the conclusion that random uncertainties dominate the Mössbauer COG test method.

\section{Discussion}

An important step in the establishment of a new metrological method is a prenormative interlaboratory study, designed to verify and validate that method against its stated aims. Here, the method being scrutinized was the Mössbauer COG method for quantifying the magnetite/maghemite composition of ironoxide-based magnetic nanoparticles.

Room temperature Mössbauer data were recorded in 7 laboratories on 6 samples chosen to constitute both a verification set of microcrystalline mixtures with a predetermined magnetite/maghemite composition, and a validation set of deliberately challenging nanoparticulate samples of unknown composition (figure 1). Initial comparisons of the as-collected raw data (figure 2) showed generally good agreement, albeit with variabilities related to: the amount of non-resonant background counts; the chosen velocity scale limits; and the statistical quality of the spectra. It was not known a priori whether these differences would significantly affect the analytical process that followed.

Data analysis proceeded both in-house and ex post facto. To maintain consistency, none of the spectra were corrected or altered in any way prior to either in-house analysis or single-operator re-analysis. Reasonable agreement was found between the $\alpha$ values obtained for all 6 samples by the different laboratories, and, for the verification samples, with the 
predetermined $\alpha$ values (figure 3). For the nanoparticles, it was found that those laboratories that were able to record low temperature spectra (for which the line profiles were sharper, and the doublets resolved into sextets, figure S3) obtained $\alpha$ values consistent with those obtained from the room temperature spectra, but there was no obvious improvement in measurement accuracy (figure 3).

Repeatability analysis on the room temperature data was undertaken using a combination of Mandel's consistency statistic (the $h$ statistic) and an adapted version of the twosample Youden plot. The $h$ values for both the mixtures and the nanoparticles (figure 4) were bench-marked against the measured $\bar{\alpha}$ means across all the participating laboratories. All but one of the measured $h$ values from the mixtures (figure 4(a)) were found to lie within the $0.5 \% t$ test significance level, and two-thirds of the $h$ values lay within \pm 1 standard deviation of the true values, indicating good accuracy and repeatability. It was also seen that although there were some differences between the $h$ values between data analysed in-house and then later by an independent operator, there were no discernible trends in those changes, and the overall spread in $h$ values was not significantly altered. All but one of the $h$ values were found to lie within the $0.5 \% t$ test significance level, and two-thirds within \pm 1 standard deviation of the measured means, indicating good repeatability at the same level as that seen for the mixtures. Again, no discernible trends were seen between the $h$ values determined using in-house versus independent-operator spectrum analysis.

Pairwise two-sample Youden-style plots were used to visualise the Mandel $h$ statistics data, and to help identify the contributions of both random and systematic uncertainties to the measurement uncertainty. The data from the mixtures and the nanoparticles was pooled, allowing 105 pairwise comparisons to be made for each of the in-house analysis and single-operator analysis sets of data (figure 5). In both cases the Youden plots indicated that apart from a few outliers, the majority (ca 92\%) of the derived data points lay inside circles of radius corresponding to the $0.5 \% t$ test significance level. As such, the Youden plots indicated both good repeatability and the dominance of random, as opposed to systematic, uncertainties.

\section{Conclusion}

Based on the prenormative study data presented in this work, we consider that the ${ }^{57} \mathrm{Fe}$ Mössbauer spectrometric 'centre of gravity' COG method is a promising candidate for an ISO standard method for determining the composition of magnetite/maghemite nanomaterials. We found that random measurement uncertainties, as opposed to systematic uncertainties, predominated, which indicates that the method is generally applicable. There was also evidence that, for the most part, these random uncertainties fell within acceptable (ASTM-standard 0.5\%-significance) limits. Furthermore, there was a very good agreement between the participating laboratories, despite the disparity in the hardware and software used. This reflects the robustness of the experimental methodology, with the widespread adoption of best practice methods in key aspects of the measurements, such as the calibration methods employed, and the use of symmetrical waveform drives and folded spectra to eliminate baseline curvature effects. For these reasons, we propose that the COG method should be considered for normalisation as a standard method for quantification of the magnetite/maghemite ratio in iron-oxide-based magnetic nanoparticles.

\section{Acknowledgments}

This work was supported in part by the European Commission Seventh Framework Programme through the NanoMag project, Grant No. 604448. In addition, MS and JZ acknowledge support from the National Science Center of Poland (2014/14/E/ST3/00026).

\section{ORCID iDs}

Lara K Bogart (D) https://orcid.org/0000-0001-7108-3980

Jeppe Fock (D) https://orcid.org/0000-0002-7515-4026

Geraldo M da Costa (D) https://orcid.org/0000-0002-13037465

Jean-Marc Greneche (D) https://orcid.org/0000-0001-73098633

Marcin Sikora (D) https://orcid.org/0000-0003-4491-3496

Mikkel Fougt Hansen (D) https://orcid.org/0000-0003-33332856

Cathrine Frandsen (D) https://orcid.org/0000-0001-5006-924X Quentin A Pankhurst (D) https://orcid.org/0000-0001-74313411

\section{References}

[1] Source: Clarivate analytics web of science (all databases) bibliographic database (accessed 21 December 2020) search for the keywords magnetic AND iron oxide AND nanoparticle OR nanomaterial under the TS heading.

[2] Pankhurst Q A, Connolly J, Jones S K and Dobson J 2003 Applications of magnetic nanoparticles in biomedicine J. Phys. D: Appl. Phys. 36 R167-81

[3] Pankhurst Q A, Thanh N K T, Jones S K and Dobson J 2009 Progress in applications of magnetic nanoparticles in biomedicine J. Phys. D: Appl. Phys. 42224001

[4] Belanova A A, Gavalas N, Makarenko Y M, Belousova M M, Soldatov A V and Zolotukhin P V 2018 Physicochemical properties of magnetic nanoparticles: implications for biomedical applications in vitro and in vivo Oncol. Res. Treat. 41 139-43

[5] Cardoso V F, Francesko A, Ribeiro C, Bañobre-López M, Martins P and Lanceros-Mendez S 2018 Advances in magnetic nanoparticles for biomedical applications $A d v$. Healthcare Mater. 71700845

[6] Rojas J M, Sanz-Ortega L, Mulens-Arias V, Gutiérrez L, Pérez-Yagüe S and Barber D F 2016 Superparamagnetic iron oxide nanoparticle uptake alters M2 macrophage phenotype, iron metabolism, migration and invasion Nanomedicine 12 1127-38

[7] Zanganeh S et al 2016 Iron oxide nanoparticles inhibit tumour growth by inducing pro-inflammatory macrophage polarization in tumour tissues Nat. Nanotechnol. 11 986-94 
[8] Gao L, Fan K and Yan X 2017 Iron oxide nanozyme: a multifunctional enzyme mimetic for biomedical applications Theranostics 7 3207-27

[9] Rusevova K, Kopinke F-D and Georgi A 2012 Nano-sized magnetic iron oxides as catalysts for heterogeneous Fentonlike reactions-influence of $\mathrm{Fe}(\mathrm{II}) / \mathrm{Fe}$ (III) ratio on catalytic performance J. Hazard. Mater. 241-242 433-40

[10] Park E-J, Umh H N, Choi D-H, Cho M H, Choi W, Kim SW, Kim Y and Kim J-H 2014 Magnetite- and maghemiteinduced different toxicity in murine alveolar macrophage cells Arch. Toxicol. 88 1607-18

[11] Daou T J, Begin-Colin S, Grenèche J M, Thomas F, Derory A, Bernhardt P, Legaré P and Pourroy G 2007 Phosphate adsorption properties of magnetite-based nanoparticles Chem. Mater. 19 4494-505

[12] Greneche J-M 2013 Mössbauer Spectroscopy: Tutorial Book ed Y Yoshida and G Langouche (Berlin: Springer) pp 187-241

[13] da Costa G M, Blanco-Andujar C, De Grave E and Pankhurst Q A 2014 Magnetic nanoparticles for in vivo use: a critical assessment of their composition J. Phys. Chem. B 118 $11738-46$

[14] Fock J, Bogart L K, Posth O, Hansen M F, Pankhurst Q A and Frandsen C 2016 Uncertainty budget for determinations of mean isomer shift from Mössbauer spectra Hyperfine Interact. 237 1-11

[15] Fock J, Bogart L K, González-Alonso D, Espeso J I, Hansen M F, Varón M, Frandsen C and Pankhurst Q A 2017 On the centre of gravity method for measuring the composition of magnetite/maghemite mixtures, or the stoichiometry of magnetite-maghemite solid solutions, via ${ }^{57} \mathrm{Fe}$ Mössbauer spectroscopy J. Phys. D: Appl. Phys. 50 265005

[16] Bogart L K, Blanco-Andujar C and Pankhurst Q A 2018 Environmental oxidative aging of iron oxide nanoparticles Appl. Phys. Lett. 113133701

[17] Contact details for 42 national representatives may be found on the Directory page of the Mössbauer Effect Data Center website www.medc.dicp.ac.cn

[18] Doublet lines should be of equal area; in sextets the area ratios of the outermost:intermediate:innermost pairs should be 3:2:1.

[19] Gorski C A and Scherer M M 2010 Determination of nanoparticulate magnetite stoichiometry by Mossbauer spectroscopy, acidic dissolution, and powder x-ray diffraction: a critical review Am. Mineral. 95 1017-26

[20] Wells J, Kazakova O, Posth O, Steinhoff U, Petronis S, Bogart L K, Southern P, Pankhurst Q and Johansson C 2017 Standardisation of magnetic nanoparticles in liquid suspension J. Phys. D: Appl. Phys. 50383003
[21] ISO/TS 19807-1:2019 Nanotechnologies-magnetic nanomaterials-part 1: specification of characteristics and measurements for magnetic nanosuspensions; and ISO/DTS 19807-2 Nanotechnologies - magnetic nanomaterials_- part 2: specification of characteristics and measurement methods for nanostructured magnetic beads for nucleic acid extraction.

[22] In ${ }^{57} \mathrm{Fe}$ Mössbauer spectrometry energy is expressed in units of $[\mathrm{v}]=\mathrm{mm} \mathrm{s}^{-1}$, where $\mathrm{v}$ is the relative velocity of a moving ${ }^{57}$ Co source foil relative to a stationary absorber. This energy may be expressed in Joules via the Doppler shift relationship, (v/c) $\mathrm{E} \gamma$, where $\mathrm{c}$ is the speed of light, and $\mathrm{E} \gamma=14.4 \mathrm{keV}=$ $2.31 \times 10^{-18} \mathrm{~J}$ is the energy of the Mössbauer $\gamma$-ray.

[23] Efforts have included the European Commission Seventh Framework Programme NanoMag project (no. 604448, 2013-17, www.nanomag-project.eu); and the European Metrology Programme for Innovation and Research MagNaStand project (no. 16NRM04, 2017-20, www. magnastand.eu)

[24] ASTM International 2018 ASTM E691-18 Standard Practice for Conducting an Interlaboratory Study to Determine the Precision of a Test Method (West Conshohocken, PA: ASTM International)

[25] Sample NM-D had the Micromod stock no. MM-06-01 and was made in January 2015; NM-E was no. MM-07-01, made in October 2015. Both were originally fluid suspensions, and were freeze dried in February 2017.

[26] Frandsen C, Ostenfeld C, Xu M, Jacobsen C, Keller L, Lefmann $\mathrm{K}$ and Mørup S 2004 Interparticle interactions in composites of nanoparticles of ferrimagnetic $\left(\gamma-\mathrm{Fe}_{2} \mathrm{O}_{3}\right)$ and antiferromagnetic $(\mathrm{CoO}, \mathrm{NiO})$ materials Phys. Rev. B 70134416

[27] Preston R S and Gerlach R 1971 Mössbauer effect in dilute alloys of iron in aluminum Phys. Rev. B 3 1519-26

[28] Margulies S, Debrunner P and Frauenfelder H 1963 Transmission and line broadening in the Mössbauer effect. II Nucl. Instrum. Methods 21 217-31

[29] Henry M, Teillet J and Varret F 1980 Effets d'épaisseur et de polarisation dans les spectres d'absorption Mössbauer; essai d'une formule simple Rev. Phys. Appl. 15 1095-102

[30] Lagarec K and Rancourt D G 1997 Extended Voigt-based analytic lineshape method for determining $\mathrm{N}$-dimensional correlated hyperfine parameter distributions in Mössbauer spectroscopy Nucl. Instrum. Methods Phys. Res. B 129 266-80

[31] Youden W J 1972 Graphical diagnosis of interlaboratory test results J. Qual. Technol. 4 29-33

[32] Mandel J 1961 Non-additivity in two-way analysis of variance J. Am. Stat. Assoc. $\mathbf{5 6} 878-88$

[33] Mandel J 1991 The validation of measurement through interlaboratory studies Chemometr. Intell. Lab. Syst. 11 109-19 